\title{
Realidade virtual nas técnicas da Terapia Cognitivo- Comportamental: Transtornos de Traumas, Ansiedade e Depressão
}

\section{Virtual reality in Cognitive Behavior Therapy techniques: Trauma Disorders, Anxiety and Depression}

\section{Realidad virtual en las técnicas de Terapia Cognitivo Comportamental: Transtornos de Traumas, Ansiedad y Depresión}

\section{Priscila Flores Prates*}

Universidade Federal de Santa Maria - UFSM, Santa Maria, Rio Grande do Sul, Brasil

\section{Alysson Oliveira Pacheco** \\ Universidade Federal de Santa Maria - UFSM, Santa Maria, Rio Grande do Sul, Brasil}

\section{Bruna Staevie dos Santos***}

Universidade Federal de Santa Maria - UFSM, Santa Maria, Rio Grande do Sul, Brasil

\section{Renan Meirelles da Silva****}

Universidade Federal de Santa Maria - UFSM, Santa Maria, Rio Grande do Sul, Brasil

\section{Raul Corrêa Ferraz*****}

Universidade Federal de Santa Maria - UFSM, Santa Maria, Rio Grande do Sul, Brasil

\section{Silvio J osé Lemos Vasconcelos******}

Universidade Federal de Santa Maria - UFSM, Santa Maria, Rio Grande do Sul, Brasil

\section{RESUMO}

Este artigo tem como objetivo investigar o que a literatura PSI traz sobre as técnicas cognitivo-comportamental, com ênfase nos Transtornos relacionados à Trauma e a Estressores, Depressivos e de Ansiedade, aliadas à realidade virtual. Este estudo foi realizado por meio de uma revisão sistemática de artigos dos últimos 10 anos nas bases de dados Scielo, Pepsic, Lilacs, Portal Capes e Psyclnfo. A busca foi feita nas línguas Portuguesa e Inglesa, totalizando 83 artigos sem filtragem e eliminando as repetições. Depois, os artigos foram filtrados de acordo com o objetivo do 
Priscila Flores Prates, Alysson Oliveira Pacheco, Bruna Staevie dos Santos, Renan Meirelles da Silva, Raul Corrêa Ferraz, Silvio J osé Lemos Vasconcelos

estudo e 14 artigos foram selecionados para a discussão neste trabalho. Como conclusão, pôde-se perceber que a realidade virtual está contribuindo para o aprimoramento e a inovação das técnicas cognitivo-comportamentais nos transtornos pesquisados. Essa junção da tecnologia e psicoterapia tem se mostrado efetiva nos resultados com os pacientes. Foi, também, observada a necessidade de mais estudos empíricos sobre o tema.

Palavras-chave: virtualidade, comportamento, depressão, ansiedade, trauma.

\begin{abstract}
This article aims to investigate what PSI literature brings on cognitive behavioral techniques allied to virtual reality with emphasis on trauma and stressor disorders, as well as depressive and anxiety disorders. This study was conducted through a systematic review of articles from the last 10 years in SciELO, Pepsic, Lilacs, Portal Capes and Psyclnfo databases. The search was conducted in Portuguese and English, totaling 83 articles without filtering and by eliminating repetitions. After that, the articles were filtered according to the purpose of the study and 14 articles were selected for this work. In conclusion, it could be seen that virtual reality is contributing to the improvement and innovation of cognitive behavior techniques in the surveyed disorders. This combination of technology and psychotherapy has proven effective in patient outcomes. It was also observed the need for more empirical studies on the subject.
\end{abstract}

Keywords: virtual, behavior, depression, anxiety, trauma.

\title{
RESUMEN
}

Este artículo tiene por objetivo investigar lo que la literatura PSI trae sobre las técnicas cognitivo comportamentales, con énfasis en los Transtornos relacionados a Trauma y a Estresores, Depresivos y de Ansiedad, aliadas a la realidad virtual. Este estudio fue realizado por medio de una revisión sistemática de arículos de los últimos 10 años en las bases de datos Scielo, Pepsic, Lilacs, Portal Capes y Psyclnfo. La búsqueda fue hecha en lengua Portuguesa e Inglesa, totalizando 83 artículos sin filtro y eliminando las repeticiones. Luego, los artículos fueron filtrados de acuerdo al objetivo del estudio y 14 articulos fueron seleccionados para la discusión en este trabajo. Como conclusión, se puede percibir que la realidad virtual está contribuyendo para el aprimoramiento y la innovación de las técnicas cognitivo comportamentales en los transtornos investigados. Esa conjunción entre tecnología y psicoterapia se ha mostrado efectiva en los resultados con los pacientes. Fue observada, también la necesidad de más estudios empíricos sobre el tema.

Palabras clave: virtualidade, comportamento, depresión, ansiedade, trauma.

\section{Introdução}

A Terapia Cognitivo-Comportamental (TCC) é composta por diferentes técnicas que combinam a abordagem cognitiva e um conjunto de procedimentos comportamentais. A TCC é utilizada como um termo mais abrangente incluindo tanto a Terapia Cognitiva padrão quanto combinações não teóricas de estratégias cognitivas e comportamentais (Knapp \& Beck, 2008). 
Na TCC, a mudança cognitiva gera a mudança comportamental e vice-versa, por isso, utiliza-se uma gama de diferentes técnicas. Essas técnicas deverão ser usadas dependendo do perfil cognitivo do transtorno e da conceitualização cognitiva do caso. No transtorno de ansiedade, por exemplo, inicialmente poderá ser aplicado o modelo cognitivo, pois há a necessidade do paciente ter um entendimento dos princípios fundamentais e, a seguir, as técnicas comportamentais. Já no transtorno depressivo, primeiramente, há a necessidade de aplicação das técnicas comportamentais a fim de promover a ativação comportamental do paciente (Knapp \& Beck, 2008).

Atualmente, tem sido percebido um movimento de introdução das técnicas cognitivo-comportamentais junto à realidade virtual (Wendt, 2011). Observa-se um grande desenvolvimento da tecnologia e o avanço desta para a contribuição em áreas como a saúde. A inovação decorrente da utilização da realidade virtual com as técnicas de psicoterapia pode ser comprovada desde que foram noticiados os primeiros casos de sucesso e de intervenções bem sucedidas valendo-se da referida tecnologia com propósitos experimentais no tratamento de fobias.

Para Dewes e colaboradores (2010), transtornos do humor e de ansiedade apresentam uma prevalência significativa em algumas parcelas da sociedade e necessitam de intervenções efetivas que auxiliem no seu manejo e tratamento. Ao investigarem a adequação de tratamentos para transtornos de humor, bem como para transtorno de ansiedade, os mesmos autores obtiveram resultados indicativos de uma maior efetividade da TCC, combinada com uso de medicamentos, para o tratamento da depressão. Ao ser comparada com abordagens inovadoras, como técnicas aliadas à realidade virtual, para o tratamento de Transtorno de Estresse Pós-Traumático, a TCC evidenciou possuir um efeito melhor sobre os sintomas que perfazem o quadro (Dewes et al., 2010).

O aspecto de maior relevância no que se refere às contribuições do presente estudo refere-se ao fato do mesmo oportunizar o contato com outros textos com a mesma temática, possibilitando uma visão mais atualizada do tema em foco, considerando a revisão de trabalhos recentes nesse campo. Pode-se afirmar que essa revisão oferece uma investigação conjunta da aplicação das técnicas da TCC aliadas à realidade virtual para os transtornos distintos que são mencionados neste trabalho e que servirá como elemento novo de comparação na busca do desenvolvimento da psicoterapia e de resultados efetivamente benéficos para o paciente.

Assim, os autores investigaram o que a literatura traz sobre as técnicas cognitivo-comportamentais, focando os transtornos relacionados a trauma e a estressores, sintomas depressivos e de ansiedade, aliadas à realidade virtual. Este estudo foi realizado por 
intermédio de uma revisão sistemática de artigos dos últimos 10 anos nas bases de dados Scielo, Pepsic, Lilacs, Portal Capes e Psyclnfo com os seguintes descritores nas línguas Portuguesa e Inglesa: terapia cognitivo-comportamental, terapia cognitiva, técnicas psicológicas, psicologia, psicoterapia, transtornos mentais, técnicas cognitivo-comportamental, realidade virtual, informática, tecnologia da informação, ambiente virtual, recursos virtuais e tecnologia.

A partir da busca nas bases de dados com a utilização dos descritores mencionados, foram encontradas 83 referências, cujos resumos foram avaliados de forma independente pelos avaliadores. $\mathrm{Na}$ primeira etapa, com base nos objetivos do estudo, foram excluídos: I) resumos repetidos; II) estudos de meta-análise; e III) resumos selecionados pelo sistema de busca das bases de dados, mas que não abordavam o tema; Assim, foram selecionados 18 resumos. A segunda etapa consistiu na leitura dos textos completos e 4 foram eliminados após a aplicação dos critérios de exclusão. Permaneceram 14 artigos que foram incluídos na presente revisão.

\section{Resultados}

A presente revisão sistemática fez uso de 14 artigos. Referente aos Transtornos relacionados a Trauma e a Estressores, foram encontrados 2 artigos internacionais, entre os quais 1 estudo empírico e 1 revisão sistemática. Sobre Transtorno Depressivo foram encontrados 2 artigos internacionais e empíricos. Entre os Transtornos de Ansiedade a busca compreendeu 10 artigos, 4 sobre fobia específica, 2 sobre Ansiedade Social, 2 sobre Agorafobia, 1 sobre Transtorno do Pânico e 1 conjuntamente sobre Fobia Específica, Agorafobia e Transtorno do Pânico, sendo 7 internacionais e 3 nacionais, 8 estudos empíricos e 2 revisões sistemáticas. A pequena quantidade de artigos encontrados, principalmente em âmbito nacional, reforça ainda mais a justificativa do presente estudo e a necessidade de pesquisa sobre o tema, revelando ser um assunto inovador.

\subsection{Transtornos relacionados a trauma e a estressores}

O transtorno relacionado a trauma e a estressores segundo o DSM-V (American Psychiatric Association, 2013) inclui transtornos nos quais a exposição a um evento traumático ou estressante está listada explicitamente como um critério diagnóstico e um dos transtornos que ele abrange é o Transtorno de Estresse Pós-Traumático. Os critérios para o TEPT, segundo o DSM V, para pessoas maiores de 6 anos de idade são: exposição a episódio concreto ou ameaça de morte, lesão grave ou violência sexual; presença de um (ou mais) 
sintomas intrusivos associados ao evento traumático, começando depois de sua ocorrência; evitação persistente de estímulos associados ao evento traumático, começando após a ocorrência do evento; alterações negativas em cognições e no humor associadas ao evento traumático começando ou piorando depois da ocorrência de tal evento; alterações marcantes na excitação e na reatividade associadas ao evento traumático, começando ou piorando após o evento; a perturbação dos critérios anteriores dura mais de um mês; a perturbação causa sofrimento clinicamente significativo e prejuízo social, profissional ou em outras áreas importantes da vida do indivíduo; e a perturbação não se deve aos efeitos fisiológicos de uma substância ou a outra condição médica.

$\mathrm{Na}$ pesquisa empírica de Freeman e colaboradores (2014), desenvolveu-se um sistema de realidade virtual para avaliar a paranoia e os sintomas de estresse pós-traumático sem a necessidade de recorrerem às lembranças. A ideia é colocar a pessoa num ambiente virtual tridimensional, em situações sociais neutras, para avaliar se há a ocorrência de erros de interpretação dos avatares como hostis.

Os participantes foram testados novamente após um período de seis meses. Os métodos utilizados pesquisa referenciada foram: no curso de um ano, 106 indivíduos foram recrutados. Os critérios de inclusão foram: ter sido atacado com uso de violência física no último mês; ter sido atendido num serviço de emergência de um hospital por lesões; idade entre 18 e 65 anos; e estar disponível para uma avaliação padrão entre 4 e 6 semanas após o ataque. Como critério de exclusão: o ataque seguir-se com abuso; o indivíduo ter histórico de doença mental e/ou dependência de drogas e álcool. A amostra final foi composta de 94 participantes. Os instrumentos para a paranoia que foram usados: Positive and Ngative Symptom Scale (PASS); Green Paranoid Thoughts Scale (GPTS); VAS paranoia, teste de autorrelato. Para o Estresse pós-traumático foram usados: Interview version of the symptoms of posttraumatic stress disorder (PTSD) symptom scale (PSSI); e pos traumatic diagnostic scale (PDS). A realidade virtual compreendeu uma viagem virtual de 4 minutos entre duas paradas do metrô de Londres com uma população de avatares. Alguns avatares interagiram com os participantes, olhando em sua direção e um avatar sorria eventualmente para ele. Para avaliar a paranoia em ambiente virtual, foi desenvolvida a escala SPSS (State Social Paranoia Scale). Para avaliar o estresse pós-traumático em ambiente virtual, foi usado o VR PTSD.

O estudo concluiu que a ocorrência de pensamentos paranoides e sintomas de estresse pós-traumático em ambiente virtual antecipam a ocorrência de problemas psiquiátricos dessa natureza em até seis meses da avaliação padrão. Isso sugere que a realidade virtual pode 
ser útil para melhor avaliar tais casos, especialmente quando não se tem certeza se o medo é infundado (Freeman et al., 2014).

O estudo de Gonçalves, Pedrozo, Coutinho, Figueira e Ventura (2012) procurou demonstrar que o senso de presença propiciado pelo ambiente virtual, rico em estímulos sensoriais, facilita 0 processamento emocional de memórias ligadas ao trauma. Essa mesma pesquisa constituiu-se por intermédio de uma revisão sistemática nos seguintes bancos de dados: ISI/Web of Knowledge, PUBMED/MEDLINE, PILOTS e PsycINFO. As buscas foram realizadas em maio de 2011, usando-se os seguintes termos: "PTSD OR stress disorder" e "virtual reality". Como critérios de inclusão, considerou-se que os pacientes deveriam ter sido diagnosticados com TEPT com base nos critérios indicados no DSM-IV e foi usada a terapia cognitivo-comportamental (TCC) como tratamento, sendo a exposição realizada em ambiente de realidade virtual. Dez artigos foram considerados para a análise final.

Como conclusão do trabalho, a terapia de exposição virtual (VRET) provou ser tão eficaz quanto a terapia de exposição convencional, sendo particularmente útil no tratamento de TEPT resistente à exposição tradicional, uma vez que permite maior engajamento do paciente e, consequentemente, maior ativação da memória traumática, o que é necessário para a extinção do medo condicionado. Os autores destacaram, entretanto, que ainda há um vasto campo a ser explorado, demandando experimentos metodologicamente mais robustos e replicações desses experimentos, a padronização do tratamento e estudos com diferentes sistemas de realidade virtual (Gonçalves et al., 2012).

\subsection{Transtornos depressivos}

Conforme o DSM-V (APA, 2013), os transtornos depressivos se configuram como os que apresentam humor triste, vazio ou irritável, acompanhado de alterações somáticas e cognitivas que afetam significativamente a capacidade de funcionamento do indivíduo, nele se incluí o Transtorno Depressivo Maior. Os critérios, segundo o DSM V para o Transtorno Depressivo Maior, são: quando cinco ou mais dos seguintes sintomas enumerados abaixo estiveram presentes durante o mesmo período de duas semanas e representam uma mudança em relação ao funcionamento anterior; pelo menos um dos sintomas é humor deprimido ou perda de interesse ou prazer. São eles: humor deprimido na maior parte do dia, quase todos os dias, conforme indicado por relato subjetivo; acentuada diminuição do interesse ou prazer em todas ou quase todas as atividades na maior parte do dia, quase todos os dias; perda ou ganho significativo de peso sem estar fazendo dieta ou redução ou aumento do apetite quase todos os dias; insônia ou hipersonia quase todos os dias; agitação ou retardo 
psicomotor quase todos os dias; fadiga ou perda de energia quase todos os dias; sentimentos de inutilidade ou culpa excessiva ou inapropriada quase todos os dias; capacidade diminuída para pensar ou se concentrar, ou indecisão, quase todos os dias; e pensamentos recorrentes de morte, ideação suicida recorrente sem um plano específico, uma tentativa de suicídio ou plano específico para cometer suicídio.

Além disso, também são critérios quando os sintomas causam sofrimento clinicamente significativo ou prejuízo no funcionamento social, profissional ou em outras áreas importantes da vida do indivíduo; o episódio não é atribuível aos efeitos fisiológicos de uma substância ou a outra condição médica; a ocorrência do episódio depressivo maior não é mais bem explicada por transtorno esquizoafetivo, esquizofrenia, transtorno esquizofreniforme, transtorno delirante, outro transtorno do espectro da esquizofrenia e outro transtorno psicótico especificado ou transtorno da esquizofrenia e outro transtorno psicótico não especificado; e nunca houve um episódio maníaco ou um episódio hipomaníaco.

A pesquisa de Williams e colaboradores (2013) relatou que uma possibilidade eficaz de aumentar o acesso dos pacientes ao tratamento da TCC para depressão, baseando-se em evidências, é por meio de programas da TCC aliada à internet (Internet-based cognitive behavioral therapy, iCBT). A TCC explicitamente lida com os vieses cognitivos da depressão por meio de um processo de avaliação cognitiva e hipótese comportamental, de modo a modificar o processamento de informações impelido pela concepção (top-down), ou seja, o processamento afetado por aquilo que o indivíduo traz à situação de estímulo. Entretanto, novas pesquisas apontam que pode ser possível modificar diretamente esses vieses (processamento impelido pelo estímulo - bottom up - ou processamento afetado diretamente pelo input de estímulo) por meio de treinamentos computadorizados, conhecidos como modificação de viés cognitivo (Cognitive-Bias Modification, CBM). Os autores consideraram útil testar se ambas as abordagens poderiam ser combinadas e desenvolvidas para uso por intermédio da internet.

$\mathrm{Na}$ pesquisa anteriormente aludida os participantes do estudo foram recrutados por intermédio de um questionário na internet. Os selecionados passaram por um diagnóstico via telefone usando o Mini International Neuropsychiatric Interview Version 5.0. As 69 pessoas que assinaram um termo de consentimento foram selecionadas a partir de um processo randômico realizado por uma pessoa independente, sendo distribuídas entre um grupo de intervenção $(\mathrm{n}=$ 38) ou uma lista de espera $(n=31)$. O Inventário Beck de Depressão e Patient Health Questionnaire-9 (PHQ-9) foram os principais recursos para determinar a severidade da depressão dos participantes. O PHQ-9 é um questionário de nove itens que avalia a 
presença de cada um dos sintomas para o episódio de depressão maior, descritos no Manual Diagnóstico e Estatístico dos Transtornos Mentais (DSM-IV), como humor deprimido, anedonia e sentimentos de culpa ou inutilidade. Esse instrumento pode ser respondido em forma de autorrelato ou aplicado por um entrevistador treinado. Outras ferramentas de apoio foram utilizadas. O componente CBM-I consistiu em sete sessões de 20 minutos completadas ao longo de uma semana. O componente de ICBT em seis lições online representando a prática da TCC bem como tarefas de casa regulares e acesso a materiais suplementares. Os citados procedimentos foram feitos, na sua totalidade, sem qualquer interação presencial. Os resultados sugeriram que 0 CBM-I para depressão pode reduzir sintomas em apenas uma semana, via sete sessões de 20 minutos, sem tarefas de casa. Os resultados também demonstraram a viabilidade de integrar CBM-I nos tratamentos existentes de iCBT para Depressão (Williams et al., 2013).

No protocolo de estudo de Martínez e colaboradores (2014), os autores delinearam um experimento paralelo com dois grupos: simples cego e randomizado individualmente, comparando a eficácia da TCC assistida por computador versus o tratamento convencional para depressão em adolescentes em clínicas de atendimento primário em Santiago, Chile. O grupo que recebeu intervenção passou por oito sessões de TCC assistida por computador, além de tratamento médico, conforme descrito nas diretrizes do Plano de Acesso Universal de Garantias Explícitas (AUGE) para depressão. O grupo controle recebeu tratamento comum de clínicas de atendimento primário, também seguindo as diretrizes do AUGE para depressão. Sintomas depressivos foram avaliados usando o Inventário Beck de Depressão (BDI). Os autores concluíram que a TCC "cara-a-cara" e assistida por computador pode melhorar os efeitos terapêuticos em participantes que recebem intervenção, comparando com o grupo controle. Os autores também comentam que pode ser difícil recrutar e motivar pacientes adolescentes para participar de intervenções psicossociais, devido a fatores como horários escolares e o estigma social associado aos tratamentos de saúde mental.

\section{Transtornos de ansiedade}

Os Transtornos de Ansiedade, segundo o DSM-V (APA, 2013), incluem transtornos que compartilham características de medo e ansiedade excessivos e perturbações comportamentais. Os artigos encontrados relacionados a esse transtorno foram sobre Fobias Específicas, Agorafobia, Ansiedade Social e Transtorno do Pânico. Segundo o DSM V, os critérios para Fobia Específica são: medo ou ansiedade acentuado acerca de um objeto ou situação; o objeto ou 
situação fóbica quase invariavelmente provoca uma resposta imediata de medo ou ansiedade; o objeto ou situação fóbica é ativamente evitado ou suportado com intensa ansiedade ou sofrimento; o medo ou ansiedade é desproporcional em relação ao perigo real imposto pelo objeto ou situação específica e ao contexto sociocultural; o medo, ansiedade ou esquiva é persistente, geralmente com duração mínima de seis meses; medo, ansiedade ou esquiva causa sofrimento clinicamente significativo ou prejuízo no funcionamento social, profissional ou em outras áreas importantes da vida do indivíduo; e a perturbação não é mais bem explicada pelos sintomas de outro transtorno mental, incluindo medo, ansiedade e esquiva de situações associadas a sintomas do tipo pânico ou outros sintomas incapacitantes, objetos ou situações relacionados a obsessões, evocação de eventos traumáticos, separação de casa ou de figuras de apego, ou situações sociais.

Para Agorafobia os critérios são: medo ou ansiedade marcante acerca de duas (ou mais) das cinco situações seguintes: uso de transporte público; permanecer em espaços abertos; permanecer em locais fechados; permanecer em uma fila ou ficar em meio a uma multidão; e sair de casa sozinho. Os demais critérios são: o indivíduo tem medo ou evita essas situações devido a pensamentos de que pode ser difícil escapar ou de que o auxílio pode não estar disponível no caso de desenvolver sintomas do tipo pânico ou outros sintomas incapacitantes ou constrangedores; as situações agorafóbicas quase sempre provocam medo ou ansiedade; as situações agorafóbicas são ativamente evitadas, requerem a presença de uma companhia ou são suportadas com intenso medo ou ansiedade. E. O medo ou ansiedade é desproporcional ao perigo real apresentado pelas situações agorafóbicas e ao contexto sociocultural; o medo, ansiedade ou esquiva é persistente, geralmente durando mais de seis meses; o medo, ansiedade ou esquiva causa sofrimento clinicamente significativo ou prejuízo no funcionamento social, profissional ou em outras áreas importantes da vida do indivíduo; se outra condição médica está presente, o medo, ansiedade ou esquiva é claramente excessivo; e o medo, ansiedade ou esquiva não são mais bem explicados pelos sintomas relacionados a outro transtorno mental.

Para Ansiedade Social os critérios são: medo ou ansiedade acentuados acerca de uma ou mais situações sociais em que o indivíduo é exposto a possível avaliação por outras pessoas; o indivíduo teme agir de forma a demonstrar sintomas de ansiedade que serão avaliados negativamente; as situações sociais quase sempre provocam medo ou ansiedade; as situações sociais são evitadas ou suportadas com intenso medo ou ansiedade; o medo ou ansiedade é desproporcional à ameaça real apresentada pela situação social e o contexto sociocultural; o medo, ansiedade ou esquiva é persistente, geralmente durando mais de seis meses; o medo, 
ansiedade ou esquiva causa sofrimento clinicamente significativo ou prejuízo no funcionamento social, profissional ou em outras áreas importantes da vida do indivíduo; o medo, ansiedade ou esquiva não é consequência dos efeitos fisiológicos de uma substância ou de outra condição médica; o medo, ansiedade ou esquiva não é mais bem explicado pelos sintomas de outro transtorno mental.

Para Transtorno do Pânico os critérios são: ataques de pânico recorrentes e inesperados; pelo menos um dos ataques foi seguido de um mês (ou mais) de uma ou de ambas as seguintes características: apreensão ou preocupação persistente acerca de ataques de pânico adicionais ou sobre suas consequências e/ou uma mudança desadaptativa significativa no comportamento relacionada aos ataques; a perturbação não é consequência dos efeitos psicológicos de uma substância ou de outra condição médica; e a perturbação não é mais bem explicada por outro transtorno mental.

A pesquisa de Paiva, Cardoso e Lamounier (2007), caracterizou-se pelo estudo da fobia de dirigir automóvel e os possíveis benefícios que a realidade virtual pode proporcionar. Foi proposto a uma equipe multidisciplinar, composta por psicólogos, analistas de sistemas e projetistas de software, o desenvolvimento de um sistema de criação de rotas automotivas virtuais flexíveis que suprissem as necessidades não alcançadas pelas outras ferramentas já existentes. Entre as limitações dessas ferramentas, incluem-se 0 alto custo de implementação e consequente encarecimento do tratamento para o paciente, a baixa flexibilidade na construção das rotas a serem percorridas e a complexidade da linguagem em que os softwares são baseados, fundamentados em "scripts" específicos. Embora tais ferramentas revelem-se tecnicamente promissoras e flexíveis para esses fins mostram-se, em contrapartida, pouco intuitivas para a maioria dos psicoterapeutas.

Dessa forma, foi elaborado, para esses mesmos fins, um programa em RV por meio de ferramentas como a Interface do Sistema (para construir a rota adequada) e o Interpretador (módulo que interpreta a rota feita em 2D para gerá-la na definição 3D). O psicólogo elaborou uma variedade de elementos do trânsito, tais como rua de mão dupla ou única, se era lisa ou esburacada, possuía ou não canteiro em sua área central, construções, frequência do tráfego, clima, período do dia, dentre outras características que eram peculiares ao paciente avaliado pelo terapeuta. O paciente tinha uma visão abrangente da rota a ser percorrida em 3D como também uma visão bastante realista de dentro do automóvel (visão do motorista e do passageiro). Esse sistema trouxe dois benefícios, o primeiro foi permitir uma maior personalização desses trajetos, de acordo com a personalidade e a necessidade de cada paciente e o segundo decorreu do fato de ser relativamente simples de ser construído por um psicólogo sem muitos conhecimentos na área da computação 
gráfica, motivo dessa ferramenta sobrepor-se a outras similares (Paiva, Cardoso, \& Lamounier, 2007).

O trabalho realizado pelos autores e apresentado no artigo citado refere-se a uma proposta bem-sucedida na resolução de duas das limitações encontradas em outros programas: a dificuldade dos psicoterapeutas ao trabalhar com linguagens de programação e a inflexibilidade na criação de rotas específicas. Entretanto, o artigo em questão não apresentou evidências empíricas ou experimentais referentes ao uso do equipamento no tratamento propriamente dito da fobia de dirigir. Diante dessas considerações, os autores também sugeriram aperfeiçoamentos no sistema, a exemplo de melhorias na interface do programa, no ambiente virtual e na avaliação dos sintomas e respostas do paciente, inclusive por meio de biofeedback, permitindo a medição de frequência cardíaca, respiração e do nível de ansiedade.

Ainda com relação à fobia de dirigir, Costa, Carvalho e Nardi (2010) tiveram como meta do trabalho investigar evidências a partir de vários estudos feitos e comprovar a eficácia da Terapia de Exposição por Realidade Virtual no tratamento desse quadro. Para tanto, foram utilizadas algumas bases de dados tais como PubMed, Medline, Web of Science e Scielo, dentro do período de 1984 a 2007. Evidenciou-se que a quantidade de estudos clínicos randomizados foi diretamente proporcional a uma maior comprovação da eficácia de TERV em transtornos de ansiedade. A grande maioria dos estudos comprovou que, entre o início e o fim do tratamento, os níveis de evitação ao estímulo temido e, consequentemente, a ansiedade diminuíram com o uso da Terapia de Exposição por Realidade Virtual. O que se destacou nesse trabalho é que a TERV pôde ser usada conjuntamente com outras técnicas, servindo como um primeiro passo no tratamento da fobia de dirigir, uma vez que facilitou a exposição ao vivo e evitou os riscos e os elevados custos dessa exposição.

O trabalho de Mühlberger, Bülthoff, Wiedemann e Pauli (2007) avaliou pessoas com fobia de túneis em relação a um grupo controle, dirigindo em três diferentes ambientes de realidade virtual (ambiente aberto, túnel semiaberto - galeria e túnel fechado). O medo de passar em túneis, tanto como motorista quanto passageiro, foi avaliado separadamente com o Fear of Tunnels Questionnaire (TAF). A pesquisa usou ainda o General Fear of Tunnels Questionnaire, que inclui uma escala de evitação de túneis; o BDI; Fear and general symptoms questionnaire, escala que contém sete tipos de situações comumente descritas como fóbicas; Symptom Checklist -90Revised, que avalia o impacto total dos sintomas no bem-estar; e o Subjetive unitsofdiscomfort, usado para avaliar as respostas de medo. Os participantes foram imersos em um ambiente virtual tridimensional com gráficos computadorizados de última geração. Os resultados mostraram que participantes com fobia de túneis 
apresentaram medo significativamente superior ao do grupo controle, que não apresentou medo substancial durante a simulação de direção em túneis. Além disso, as respostas de medo somente variaram dentro dos três tipos de ambiente virtual no grupo de pessoas com fobia de túneis - quanto mais fechado o ambiente maior o medo. Houve ainda um aumento do medo ao entrar no túnel e um decréscimo ao sair.

Vários estudos sobre tratamento do medo de altura (acrofobia) foram feitos. Coelho, Pocinho e Silva (2008) fizeram comparativos entre intervenções que apresentaram algumas semelhanças com sua própria experiência clínica e de investigação com o objetivo de demonstrar as estratégias terapêuticas que julgaram mais apropriadas ao tratamento do medo de alturas. Na investigação das diversas práticas, cujo objetivo foi a avaliação e tratamento da acrofobia, constatou-se que a prática reforçada foi a que se mostrou mais estudada e com garantias de melhores resultados (Leitenberg, 1976). A finalidade da mesma no tratamento de fobias foi a diminuição da evitação do estímulo ou situação temida e a moldagem de comportamentos de aproximação apropriados. Nessa prática, o terapeuta proporciona ao paciente uma exposição gradual ao alvo ameaçador e reforça melhorias sucessivas à habilidade do paciente para interagir com o estímulo alvo. Variáveis como o elogio, 0 feedback preciso, o conhecimento do desempenho e as expectativas do paciente e do terapeuta também são elementos importantes na terapia.

Em um estudo precursor ao uso de sistemas virtuais citado pelos autores, Schneider (1982) usou binóculos com lentes invertidas para alterar a percepção de profundidade, de forma a magnificar a sensação de altura durante um processo de exposição ao vivo. A volta do paciente 8 meses após o término do tratamento se mostrou positiva, avaliando a manutenção dos efeitos da referida intervenção (Coelho, Pocinho, \& Silva, 2008). Atualmente, a realidade virtual (RV) possui várias aplicações no campo da Psicologia. Em outro artigo, Coelho, Silva, Santos \& Silvério (2005), sabendo que a exposição ao vivo é eficaz para indivíduos com medo de altura, investigaram se esse tipo de tratamento pode ser efetuado através de sistemas de RV. Com o método de estudo de caso, constatou-se que após 3 sessões de exposição a alturas simuladas em ambiente virtual o paciente apresentou progressos significativos nas medidas de ansiedade e evitação quando confrontado com situações reais. Conclui-se que os principais elementos do tratamento de exposição encontram-se bem fundamentados na prática reforçada de Leitenberg (1976), quer em ambientes reais, quer em ambientes de realidade virtual.

No estudo realizado por Price e Anderson (2012), foram avaliados os efeitos das expectativas de resultados como um preditor de resposta 
ao tratamento para medo de falar em público por meio da realidade virtual individual e as terapias cognitivo-comportamentais em grupo. A pesquisa partiu da hipótese de que uma expectativa positiva inicial acerca do tratamento resultaria em uma melhor resposta a ele, tanto no que concerne à realidade virtual quanto à terapia em grupo. Para critério de inclusão foi definido o diagnóstico de ansiedade social com medo primário de falar em público, sendo avaliado a partir do diagnóstic and statistical manual of mental disorders - fourth edition (DSM-IV; APA, 2000). A amostra total compreendeu 67 participantes que estavam com a medicação estabilizada por pelo menos 3 meses. Os participantes responderam dois instrumentos de autorrelato que avaliavam a ansiedade social, the Personal Report of Communication Apprehension-Short Form (PRCA-SF; McCroskey, 1978) e the Self Statements during Public Speaking (SSPS; Hofmann \& DiBartolo, 2000), além de um terceiro questionário que avaliou as expectativas acerca do tratamento. Os resultados apoiaram o efeito das expectativas de resultados como um preditor da taxa de mudança na ansiedade de falar em público tanto durante a terapia de exposição realidade virtual individual quanto na terapia comportamental em grupo cognitivo. Além disso, não houve evidência para sugerir que o impacto da expectativa de resultado era diferente na realidade virtual ou nos tratamentos em grupo.

O artigo de Anderson, Zimand, Hodges e Rothbaum (2005), destaca a dificuldade de se fazer exposição com pessoas com fobia de falar em público. Assim, a realidade virtual se torna uma ferramenta alternativa que já apresentou bons resultados em dois estudos de caso. A amostra foi composta por dez pessoas, oito com fobia social e duas com síndrome do pânico com agorafobia, em que o medo de falar em público é o estimulo mais temido. Os participantes receberam tratamento individual por oito sessões. O tratamento consistia em 4 sessões para controle e gerenciamento da ansiedade (controle da respiração, reestruturação cognitiva, e experimentos comportamentais para desafiar as crenças) e quatro sessões de exposição através de realidade virtual. O ambiente da realidade virtual mostrava cinco pessoas ao redor de uma mesa de conferência ou vinte e duas em um auditório. O participante tinha que discursar para as pessoas virtuais em um ambiente de alta definição, enquanto os terapeutas controlavam as reações das pessoas do ambiente virtual e podiam se comunicar com o participante usando um microfone. Os scores de todas as medidas de fobia em falar em público melhoraram significativamente do pré-tratamento para o póstratamento e esses ganhos foram mantidos com o passar do tempo. A terapia cognitiva comportamental usando a exposição por meio da realidade virtual foi eficiente no tratamento. Os participantes ficaram satisfeitos e houve manutenção dos ganhos ao passar do tempo. 
O estudo de Anderson e colaboradores (2006) examinou se, a longo prazo, para o medo de voar, houve manutenção dos ganhos do tratamento com terapia cognitivo-comportamental após os ataques terroristas de 11 de setembro. Foram realizados tanto o tratamento de exposição padrão quanto um tratamento de exposição baseado na realidade virtual. Para participar da pesquisa, os indivíduos deviam se encaixar nos critérios do DSM-IV para fobia específica (do tipo fobia de voar), síndrome do pânico, em que o medo de voar está como um dos estímulos temidos ou agorafobia, sem síndrome do pânico, em que o medo também se apresenta em relação a voar. Participaram do tratamento e da pesquisa inicial 115 pessoas. Desse total, 55 completaram a pesquisa subsequente, realizada após 11 de setembro, resultando em $48 \%(55 / 115)$ de retenção. Foram usados o FFI (The Fear of Flying Inventary) e o QAF (Questionnaire on Atitudes Toward Flying). Após o tratamento, os participantes foram contatados e questionados sobre quantos voos haviam feito no decorrer dos últimos seis meses. Após 11 de setembro, os participantes foram questionados quantos voos fizeram desde o episódio (o tratamento e a pesquisa começaram antes de 11 de setembro e após esse episódio, os pesquisadores optaram por analisar também o impacto do evento sobre o tratamento).

Os participantes foram designados aleatoriamente para receber ou o tratamento de exposição padrão ou o tratamento de exposição baseado em realidade virtual por oito sessões individuais durante seis semanas. O tratamento de exposição padrão foi realizado numa aeronave real, estacionada. Os resultados sugerem que indivíduos tratados com terapia cognitivo-comportamental de curto prazo mantiveram os ganhos a longo prazo, mesmo após o evento catastrófico de 11 de setembro. Os participantes relataram melhoras constantes nas duas escalas de autorrelato. O artigo não diferencia qual dos dois tipos foi mais eficiente, mas como os dois mantiveram os ganhos do tratamento, é possível considerar que realidade virtual mostre-se mais vantajosa em termos de custo-benefício (Anderson et al., 2006).

O Objetivo da pesquisa de Castro e colaboradores (2014) foi avaliar a eficácia do tratamento de exposição por realidade virtual dentro da abordagem da TCC, em um contexto de múltiplas exposições, combinada com a exposição in vivo para melhorar o engajamento com o tratamento e reduzir as taxas de desistência em pacientes com agorafobia de longo prazo (acima de cinco anos). Os pesquisadores objetivaram ainda comparar a eficácia do tratamento citado acima com a TCC tradicional e a monoterapia de antidepressivos. A amostra inicial contou com 80 participantes, dentre os quais 30 desistiram ao longo do processo, restando 50 pacientes com cinco ou mais anos de evolução de agorafobia (com e sem ataques de pânico) que completaram o tratamento. Para a exposição por realidade virtual, os 
pesquisadores usaram um software que permite a simulação de sete ambientes que podem desencadear estímulos fóbicos em portadores de agorafobia. Os ambientes são: um aeroporto e um avião; uma praça e uma rua; um elevador e um estacionamento subterrâneo; um banco; uma autoestrada; uma praia e um teleférico.

Como resultados, o estudo mostrou uma melhor aderência no grupo com exposição virtual, mas sem diferença estatística significativa com o grupo da TCC tradicional. Além disso, os efeitos combinados da exposição por realidade virtual mais antidepressivos mostraram melhor eficácia, considerando a melhora clínica. Os autores acreditaram que esse resultado pode se dever a uma maior capacidade de se autorregular por um processo que foi um bom intermediário (exposição virtual) antes da confrontação real com o estímulo fóbico. A aproximação com 0 estimulo real (dessensibilização sistemática) encontraria na realidade virtual um eficaz facilitador do processo. Isso pôde ser um facilitador do tratamento para pessoas com agorafobia de longo-prazo, porque essas pessoas costumam ter histórico de fracassos quando expostas ao estímulo fóbico real. Outra suposição decorrente do trabalho em questão, foi a de que a realidade virtual permitiu mudanças cognitivas, aumento do senso de autoeficácia e autoafirmação (Castro et al. 2014).

Para Vincelli e Riva (2002), todo o processo de tratamento para o transtorno do pânico e agorafobia proposto recebia o nome de Terapia Experencial-Cognitiva, a qual fazia a integração do uso de Realidade Virtual (RV), objetivando descondicionar reações de medo para modificar a cognição interpretacional relacionada com os sintomas de pânico e consequente redução da ansiedade. O uso da RV oferecia um significativo aumento de eficácia terapêutica por diversos fatores. Infere-se que o principal deles pode estar relacionado ao fato de que, em tais condições, o paciente não temia que algo realmente acontecesse com ele, pois sabia que estava em um ambiente virtual. Com essa certeza, os participantes podiam explorar livremente sentimentos e/ou pensamentos de experiência. Um fato que pode ter tornado cada paciente mais apto ao enfrentamento de situações recorrentes no mundo real. Além desse fator, considera-se o fato de que a concentração que se mostrou possível para os sujeitos envolvidos na experiência virtual era significativamente maior do que em grupos de controle tratados in vivo. A abordagem com RV, além de eficaz, evidenciava diversas vantagens, como por exemplo, o fato de que o psicólogo controlava todos os parâmetros implicados na terapia individual de cada paciente.

O objetivo do estudo de De Carvalho, Freire e Nardi (2008) foi realizar uma revisão sistemática da literatura sobre realidade virtual e psicoterapia com ênfase na técnica de exposição, componente já 
importante da terapia cognitivo-comportamental e em estudos que enfocam o tratamento do transtorno de pânico (TP). A busca foi realizada na base de dados PubMed, por meio das palavras-chave: "virtual reality" e "panic disorder". Não houve na busca delimitação do período de publicação dos artigos e só foram selecionados artigos em língua inglesa. Foram selecionados 28 artigos.

Os achados do estudo de De Carvalho, Freire e Nardi (2008) demostraram que o grande potencial oferecido pela realidade virtual à psicologia clínica derivou do importante papel que a memória e a imaginação desempenham nos transtornos e, consequentemente, na psicoterapia. Nos transtornos ansiosos, e mais especificamente no transtorno do pânico, sabe-se que as imagens mentais ansiogênicas são responsáveis pelo ciclo de manutenção do medo, da preocupação e/ou do desconforto, resultando e interagindo com distorções cognitivas, comportamentos desadaptativos e emoções negativas. Uma técnica comportamental importante é a técnica de exposição. 0 sentimento de presença que os ambientes virtuais permitem experimentar e o envolvimento sensório-motor proporcionam uma sensação de imersão mais realista em comparação ao que poderia ser experimentado a partir da construção de cenários meramente imaginários, obtendo assim a segurança inicial para eventuais exposições ao vivo. Isso demostra os bons resultados da realidade virtual aplicada ao tratamento do TP.

\section{Discussão e considerações finais}

Ao investigar os artigos selecionados para este trabalho, observou-se que a realidade virtual está contribuindo para o aprimoramento e para a inovação das técnicas da Terapia Cognitivo-Comportamental nos Transtornos relacionados ao trauma, à depressão e à ansiedade. Essa articulação da tecnologia e psicoterapia, principalmente a Terapia Cognitivo-Comportamental, tem se mostrado efetiva nos resultados com os pacientes que buscam ajuda para o tratamento de algum destes desconfortos.

Os principais achados nos estudos pesquisados foram: sobre o transtorno de traumas e estressores especificamente o Transtorno de Estresse Pós - Traumático: o benefício do uso da realidade virtual no tratamento deste transtorno. O uso da tecnologia pode antecipar a ocorrência de problemas psiquiátricos em até 6 meses, permitindo que o paciente envolva-se mais com o processo terapêutico, tendo assim uma maior ativação da memória traumática, necessária para acabar com o medo condicionado. As pesquisas que foram analisadas sobre o transtorno depressivo maior tiveram como resultados a efetividade de técnicas e tarefas executadas por meio de instrumentos do ambiente virtual. Os estudos revisados evidenciaram 
também a redução dos sintomas de depressão e a motivação dos pacientes em aderir ao modelo de tratamento, considerando as possíveis adequações aos seus estilos de vida. Nos Transtornos de Ansiedade, os principais achados estão relacionados às fobias específicas. Observou-se que as inovações tecnológicas nos instrumentos utilizados para o tratamento dos diferentes tipos de medo, apesar de necessitarem de avanços, já resultam em pontos positivos na psicoterapia conduzida com indivíduos que foram submetidos a essas mesmas modalidades de tratamento. Sobre a ansiedade social, os estudos feitos com a realidade virtual indicaram um número maior de efeitos positivos quando comparados a intervenções grupais realizadas ao vivo. A ansiedade de falar em público teve suas taxas diminuídas, conforme indicaram alguns trabalhos revisados. No Transtorno do Pânico e Agorafobia, os resultados foram positivos e os tratamentos obtiveram sucesso principalmente pela simulação do ambiente real por intermédio da realidade virtual, fazendo com que houvesse uma redução quanto ao medo dos participantes diante de uma situação real.

Sendo assim, pode-se perceber que realidade virtual possibilita a simulação de eventos ou situações reais em um ambiente controlado e relativamente seguro, oportunizando tanto para terapeutas quanto para pacientes uma ampliação do rol de possibilidades relativas ao tratamento. Além disso, destaca-se ainda o fato de que é possível controlar uma grande parte das variáveis de relevância em um ambiente virtual, algo, na maioria das vezes, inviável quando se trata de ambientes reais.

O presente estudo possibilitou a pesquisa e compilação de vários outros estudos sobre a temática realidade virtual na terapia cognitivo-comportamental. Ressalta-se que há a necessidade de uma ampliação, em quantidade e qualidade, nas pesquisas sobre a temática abordada, pois, as psicoterapias precisam estar em um constante processo evolutivo, acompanhando tanto a natureza dinâmica das relações e experiências humanas quanto a presença cada vez mais intensa da tecnologia na vida cotidiana. A partir disso, espera-se que os pesquisadores que trabalham em tais contextos, na esfera da saúde mental, bem como aqueles que trabalham diretamente com a tecnologia, possam motivar-se para iniciar novas pesquisas, unindo cada vez mais essas áreas.

\section{Referências}

Anderson, P., Jacobs, C. H., Lindner, G. K., Edwards, S., Zimand, E., Hodges, L., \& Rothbaum, B. (2006). Cognitive Behavior Therapy for Fear of Flying: Sustainability of Treatment Gains After September 11. Behavior Therapy, 37, 91-97. 
Anderson, P., Zimand, E., Hodges, L., \& Rothbaum, B. (2005). Cognitive Behavioral Therapy for Public-Speaking Anxiety using Virtual Reality for Exposure. Depression and Anxiety, 22, 156158.

American Psychiatric Association - APA (2013). Diagnostic and Statistical Manual of Mental Disorders. 5 ed. Washington, DC.

Castro, W. P., Sanchés, M. J. R., González, C. T. P., Bethencourt, J. M., Portero, J. A. de la F., \& Marco, R. G. (2014). Cognitivebehavioral treatment and antidepressants combined with virtual reality exposure for patients with chronic agoraphobia. International Journal of Clinical and Health Psychology, 14, 917.

Coelho, C. M., Pocinho, M., \& Silva, C. F. (2008). O tratamento do medo de alturas. Psicologia Argumento, 26(53), 99-108.

Coelho, C. S. M., Silva, C. F., Santos, J. A., Silvério, J. A. S. (2005). Realidade Virtual Aplicada ao tratamento da acrofobia: Estudo de Caso. Psiquiatria Clínica, 26(2), 153-165.

Costa, R. T., Carvalho, M. R., Nardi, A. E. (2010). Virtual Reality Exposure Therapy in the Treatment of Driving Phobia. Psicologia: Teoria e Pesquisa, 26(1), 131-137.

De Carvalho, M. R., Freire, R. C., \& Nardi, A. E. (2008). Realidade virtual no tratamento do transtorno de pânico. Jornal Brasileiro de Psiquiatria, 57(1), 64-69.

Dewes, D., Oliveira, M. S., Andretta, I., Mühlen, B. K. V., Camargo, J., \& Leite, J. C. C. (2010). Efetividade da Terapia CognitivoComportamental para os Transtornos do Humor e Ansiedade: Uma revisão de Revisões Sistemáticas. Revista de Psicologia da I MED, 2(2), 385-397.

Freeman, D., Antley, A., Ehlers, A., Dunn, G., Thompson, C., Vorontsova, N., Garety, P., Kuipers, E., Gluksman, E., \& Slater, M. (2014), The use of immersive virtual reality (VR) to predict the occurrence 6 months later of paranoid thinking and posttraumatic stress symptoms assessed by self-report and interviewer methods: a study of individuals who have been physically assaulted. Psychological Assessment, 26(3), 841847.

Gonçalves, R., Pedrozo, A. L., Coutinho, E. S. F., Figueira, I., \& Ventura, P. (2012). Efficacy of Virtual Reality Exposure Therapy in the Treatment of PTSD: A Systematic Review. Plos One, 7(12): e48469.

Knapp, P., \& Beck, A. T. (2008). Fundamentos, modelos conceituais, aplicações e pesquisa da terapia cognitiva. Revista Brasileira de Psiquiatria, 30(2), 54-64.

Leitenberg, H. (1976). Behavioral approaches to treatment of neuroses. In H., Leitenberg (Ed.). Handbook of behavior 
modification and behavior therapy. Englewood Cliffs: PrenticeHall.

Lovell, K., \& Richards, D. (2000). Multiple access points and levels of entry (MAPLE): Ensuring choice, accessibility and equity for CBT services. Behavioural and Cognitive Psychotherapy, 28, 379391.

Martínez, V., Martínez, P., Vöhringer, P. A., Araya, R., \& Rojas, G. (2014). Computer assisted cognitive-behavioral therapy for adolescent depression in primary care clinics in Santiago, Chile (YPSA-M): study protocol for a randomized controlled trial. Trials, 15, 309.

Mühlberger, A., Bülthoff, H. H., Wiedemann, G., \& Pauli, P. (2007). Virtual Reality for the Psychophysiological Assessment of Phobic Fear: Responses During Virtual Tunnel Driving. Psychological Assessment, 19(3), 340-346.

National Institute for Health and Clinical Excellence. (2009). Depression: Management of depression in primary and secondary care. London, England: Author.

Paiva, J. G. S., Cardoso, A., \& Lamounier Jr., E. (2007). Uma proposta para o tratamento de fobias de direção através da criação de rotas automotivas virtuais. Aletheia, 25, 97-108.

Price, M., \& Anderson, P. L. (2012). Outcome Expectancy as a Predictor of Treatment Response in Cognitive Behavioral Therapy for Public Speaking Fears Within Social Anxiety Disorder. Psychotherapy, 49(2), 173-179.

Rude, S. S., Wenzlaff, R. M., Gibbs, B., Vane, J., \& Whitney, T. (2002). Negative processing biases predict subsequent depressive symptoms. Cognition \& Emotion, 16, 423-440.

Vincelli, F., \& Riva, G. (2002). Virtual Reality: a new tool for panic disorder therapy. Expert Rev. Neurotherapeutics, 2(3), 377383.

Wendt, G. W. (2011). Tecnologias de interface humanocomputacional: realidade virtual e novos caminhos para pesquisa. Revista de Psiquiatria Clínica, 38(5), 211-2.

Williams, A. D., Blackwell, S. E., Mackenzie, A., Holmes, E. A., \& Andrews, G. (2013). Combining Imagination and Reason in the Treatment of Depression: A Randomized Controlled Trial of Internet-Based Cognitive-Bias. Journal of Consulting and Clinical Psychology, 81(5), 793-799.

\section{Endereço para correspondência Priscila Flores Prates}

Universidade Federal de Santa Maria - UFSM

Centro de Ciências Sociais e Humanas - Departamento de Psicologia

Avenida Roraima, 1000, sala 304 (3204), Cidade Universitária, Camobi, CEP 97105900, Santa Maria - RS, Brasil 
Priscila Flores Prates, Alysson Oliveira Pacheco, Bruna Staevie dos Santos, Renan Meirelles da Silva, Raul Corrêa Ferraz, Silvio J osé Lemos Vasconcelos

Endereço eletrônico: psico_piti@yahoo.com.br

Alysson Oliveira Pacheco

Universidade Federal de Santa Maria - UFSM

Centro de Ciências Sociais e Humanas - Departamento de Psicologia

Avenida Roraima, 1000, sala 304 (3204), Cidade Universitária, Camobi, CEP 97105-

900, Santa Maria - RS, Brasil

Endereço eletrônico: alysson.o.pacheco@gmail.com

Bruna Staevie dos Santos

Universidade Federal de Santa Maria - UFSM

Centro de Ciências Sociais e Humanas - Departamento de Psicologia

Avenida Roraima, 1000, sala 304 (3204), Cidade Universitária, Camobi, CEP 97105900, Santa Maria - RS, Brasil

Endereço eletrônico: brunastaevie@gmail.com

\section{Renan Meirelles da Silva}

Universidade Federal de Santa Maria - UFSM

Centro de Ciências Sociais e Humanas - Departamento de Psicologia

Avenida Roraima, 1000, sala 304 (3204), Cidade Universitária, Camobi, CEP 97105900, Santa Maria - RS, Brasil

Endereço eletrônico: renanmeirelles@globomail.com

\section{Raul Corrêa Ferraz}

Universidade Federal de Santa Maria - UFSM

Centro de Ciências Sociais e Humanas - Departamento de Psicologia

Avenida Roraima, 1000, sala 304 (3204), Cidade Universitária, Camobi, CEP 97105900, Santa Maria - RS, Brasil

Endereço eletrônico: raulcferraz@gmail.com

Silvio J osé Lemos Vasconcelos

Universidade Federal de Santa Maria - UFSM

Centro de Ciências Sociais e Humanas - Departamento de Psicologia

Avenida Roraima, 1000, sala 304 (3204), Cidade Universitária, Camobi, CEP 97105-

900, Santa Maria - RS, Brasil

Endereço eletrônico: silviojlvasco@hotmail.com

Recebido em: 23/03/2015

Reformulado em: 13/04/2016

Aceito para avaliação em: 29/04/2016 